\title{
PERIODS OF DUCCI SEQUENCES AND ODD SOLUTIONS TO A PELLIAN EQUATION
}

\author{
FLORIAN BREUER
}

(Received 10 October 2018; accepted 30 November 2018; first published online 3 July 2019)

\section{Abstract}

A Ducci sequence is a sequence of integer $n$-tuples generated by iterating the map

$$
D:\left(a_{1}, a_{2}, \ldots, a_{n}\right) \mapsto\left(\left|a_{1}-a_{2}\right|,\left|a_{2}-a_{3}\right|, \ldots,\left|a_{n}-a_{1}\right|\right) .
$$

Such a sequence is eventually periodic and we denote by $P(n)$ the maximal period of such sequences for given $n$. We prove a new upper bound in the case where $n$ is a power of a prime $p \equiv 5(\bmod 8)$ for which 2 is a primitive root and the Pellian equation $x^{2}-p y^{2}=-4$ has no solutions in odd integers $x$ and $y$.

2010 Mathematics subject classification: primary 11B83; secondary 11D09, 11R18.

Keywords and phrases: Ducci sequence, Pellian equation, cyclotomic field, real quadratic unit.

\section{Introduction}

Let $n$ be a positive integer and consider the map $D: \mathbb{Z}^{n} \rightarrow \mathbb{Z}^{n}$ defined by

$$
D:\left(a_{1}, a_{2}, \ldots, a_{n}\right) \mapsto\left(\left|a_{1}-a_{2}\right|,\left|a_{2}-a_{3}\right|, \ldots,\left|a_{n}-a_{1}\right|\right) .
$$

Sequences of integer $n$-tuples obtained by iterating this map are known as Ducci sequences, in honour of E. Ducci, who first studied them in the 1930s. He discovered that every such sequence of integer $n$-tuples eventually stabilises at $(0,0, \ldots, 0)$ if and only if $n$ is a power of 2 (see [8]).

Ducci sequences and their generalisations have received much attention in the literature (see, for example, [4-7, 9, 11, 18]) and they have been independently rediscovered in various guises by several authors (for example in $[1-3,12,16]$ ).

Since the entries in a Ducci sequence remain bounded, the sequence eventually becomes periodic. We are interested here in the period $P(n)$ of the Ducci sequence starting with $(0, \ldots, 0,1)$. The function $P(n)$ was studied in detail in [11] and it is shown that the period of any Ducci sequence of $n$-tuples divides $P(n), n$ divides $P(n)$ and $P\left(2^{k} n\right)=2^{k} P(n)$. Thus it suffices to study $P(n)$ for odd $n$. Furthermore, one has the following upper bounds on $P(n)$.

(C) 2019 Australian Mathematical Publishing Association Inc.

This is an Open Access article, distributed under the terms of the Creative Commons Attribution licence (http://creativecommons.org/licenses/by/4.0/), which permits unrestricted re-use, distribution, and reproduction in any medium, provided the original work is properly cited. 
Theorem 1.1 [11]. Suppose $n$ is odd.

(1) Denote by $m=\operatorname{ord}_{n}(2)$ the multiplicative order of 2 modulo $n$. Then $P(n)$ divides $B_{1}(n):=2^{m}-1$.

(2) Suppose there exists an integer $M$ for which $2^{M} \equiv-1(\bmod n)$. In this case we say that ' $n$ is with -1 '. If $M$ is the smallest such integer, then $P(n)$ divides $B_{2}(n):=n\left(2^{M}-1\right)$.

In [5] we list the first few odd values of $n$ satisfying various sharpness conditions relative to the bounds in Theorem 1.1. In particular, the first examples of $n$ with -1 for which $P(n)<B_{2}(n)$ are $n=37,101,197,269,349,373,389,541,557$ and 677. Searching the Online Encyclopedia of Integer Sequences we find that, with the exception of 541, these are the first nine entries of Sequence A130229 [15]: the primes of the form $p \equiv 5(\bmod 8)$ for which the Pellian equation

$$
x^{2}-p y^{2}=-4
$$

has no solution in odd integers $x$ and $y$.

Our goal is to prove the following result, which explains this discovery.

THEOREM 1.2. Let $p \equiv 5(\bmod 8)$ be a prime such that 2 is a primitive root modulo $p$ and for which (1.1) has no solution in odd integers $x$ and $y$. Then $P(p)$ divides $\frac{1}{3} B_{2}(p)$. If furthermore $p$ is not a Wieferich prime, then $P\left(p^{k}\right)$ divides $\frac{1}{3} B_{2}\left(p^{k}\right)$ for all positive integers $k$.

Recall that an integer $a$ is a primitive root modulo $n$ if $\operatorname{ord}_{n}(a)=\varphi(n)$, that is, $a$ generates $(\mathbb{Z} / n \mathbb{Z})^{*}$. Artin's Conjecture states that every nonsquare integer $a \neq-1$ is a primitive root modulo $p$ for infinitely many primes $p$. When 2 is a primitive root modulo $n$, then $2^{\operatorname{ord}_{n}(2) / 2} \equiv-1(\bmod n)$, so $n$ is with -1 .

A prime $p$ is called a Wieferich prime if $2^{p-1} \equiv 1\left(\bmod p^{2}\right)$. Only two Wieferich primes are known, 1093 and 3511, neither of which satisfies the hypothesis of Theorem 1.2. However, a standard heuristic argument suggests that the number of Wieferich primes $p \leq x$ should grow like $\log \log (x)$ (see [4, Section 9]).

The condition that 2 is a primitive root modulo $p$ in Theorem 1.2 is essential; the first entry in sequence A130229 for which 2 is not a primitive root is 997 and in fact $P(997)=B_{2}(997)=997\left(2^{166}-1\right)$.

The case $n=541$ does not fit into our scheme; instead $P(541)=\frac{1}{7} B_{2}(541)$.

\section{Periods and cyclotomy}

It is known (see, for example, [7]) that the tuples in the periodic part of a Ducci sequence all lie in $\{0, c\}^{n}$, for some constant $c$. Therefore, after discarding the common factor $c$, we may assume that all entries lie in $\{0,1\}^{n}=\mathbb{F}_{2}^{n}$, in which case the Ducci operator $D$ becomes linear:

$$
D: \mathbb{F}_{2}^{n} \rightarrow \mathbb{F}_{2}^{n} ; \quad\left(a_{1}, a_{2}, \ldots, a_{n}\right) \mapsto\left(a_{1}+a_{2}, a_{2}+a_{3}, \ldots, a_{n}+a_{1}\right) .
$$


Next, mapping a tuple $u=\left(a_{1}, a_{2}, \ldots, a_{n}\right)$ to the element represented by the polynomial $f=a_{1} x^{n-1}+a_{2} x^{n-2}+\cdots+a_{n}$ in the ring $R=\mathbb{F}_{2}[x] /\left\langle x^{n}-1\right\rangle$, we find that the Ducci sequence $u, D u, D^{2} u, \ldots \in \mathbb{F}_{2}^{n}$ maps to the sequence $f,(x+1) f,(x+1)^{2} f, \ldots \in R$, an idea going back to [18]. We thus find that $P(n)$ equals the multiplicative period of $x+1$ in $R$. Realising $R$ to be the ring of cyclotomic integers modulo 2 , we arrive at the following result (see [4, Theorem 5.2]).

Theorem 2.1. Suppose $n$ is odd. Denote by $L=\mathbb{Q}\left(\zeta_{n}\right)$ the nth cyclotomic field, where $\zeta_{n} \in \mathbb{C}$ is a primitive nth root of unity. Denote by $O_{L}=\mathbb{Z}\left[\zeta_{n}\right]$ the ring of integers in L. Let $\mathfrak{P} \subset \mathrm{O}_{L}$ be a prime ideal containing 2. Then $P(n)$ equals the lowest common multiple of the multiplicative orders of $\zeta+1$ modulo $\mathfrak{P}$, where $\zeta$ ranges over all nth roots of unity $\zeta \neq 1$.

Since $\left(O_{L} / \mathfrak{P}\right)^{*}$ has order $B_{1}(n)$, we recover the bound $P(n) \mid B_{1}(n)$. Further, note that $\zeta+1=\left(1-\zeta^{2}\right) /(1-\zeta)$ is a unit in $O_{L}$ by [10, Proposition 3.5.5], so one source of sharper bounds for $P(n)$ arises when the units of $O_{L}$ generate a proper subgroup of $\left(O_{L} / \mathfrak{P}\right)^{*}$. Determining the units of $O_{L}$ is generally difficult, but under certain circumstances this phenomenon can be detected already at the level of a quadratic subfield $\mathbb{Q}(\sqrt{d}) \subset L=\mathbb{Q}\left(\zeta_{n}\right)$, which is where the Pellian equation (1.1) comes into play.

\section{Proof of Theorem 1.2}

Suppose that $p \equiv 5(\bmod 8)$ and that 2 is a primitive root modulo $n=p^{k}$. If $p$ is not a Wieferich prime, then this follows if 2 is a primitive root modulo $p$, by [10, Proposition 2.1.24]. Now 2 remains prime in $\mathbb{Q}\left(\zeta_{n}\right)$, that is, $\mathfrak{P}=2 O_{L}$, by [10, Proposition 3.5.18].

By [10, Propositions 3.4.1 and 3.5.14], $\mathbb{Q}\left(\zeta_{p}\right)$, and thus also $L$, contains the real quadratic field $K=\mathbb{Q}(\sqrt{p})$, whose ring of integers is $O_{K}=\mathbb{Z}[(1+\sqrt{p}) / 2]$. Let $\mathfrak{p}=\mathfrak{P} \cap K=2 O_{K}$.

Since $\mathfrak{p}$ is inert in $L / K$, we have $\operatorname{Gal}(L / K) \cong \operatorname{Gal}\left(\left(O_{L} / \mathfrak{P}\right) /\left(O_{K} / \mathfrak{p}\right)\right)$, and thus the norm $N_{L / K}: L \rightarrow K$ induces the commutative diagram

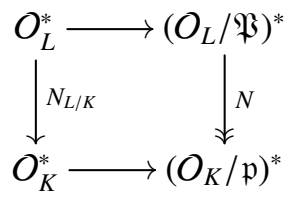

where the second vertical map is the norm of finite fields, which is surjective by [10, Proposition 2.4.12].

The group of units $O_{K}^{*}$ is generated by -1 and the fundamental unit in $K$, say $\varepsilon=(x+y \sqrt{p}) / 2$, where $(x, y)$ is the fundamental solution to (1.1), see [10, Proposition 6.3.16] and [17]. Therefore, we see that the units $O_{K}^{*}$ generate the trivial subgroup $\{1\}<\left(O_{K} / \mathfrak{p}\right)^{*} \cong \mathbb{F}_{4}^{*}$ if and only if (1.1) has no odd solutions. In this case, the image of the bottom horizontal arrow is a subgroup of index 3. It follows that the image of the top arrow lies in a subgroup of index 3 and thus $P(n) \mid \frac{1}{3} B_{1}(n)$. Since $p \equiv 1$ 


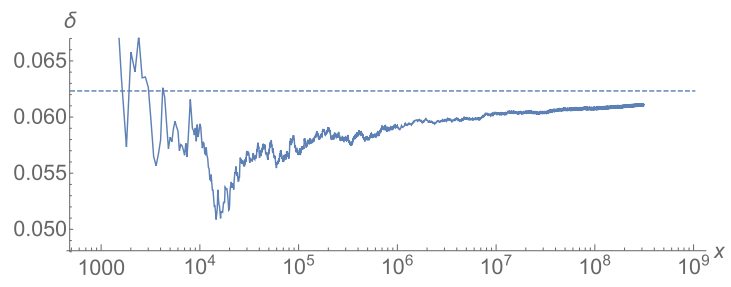

FIGURE 1. Proportion $\delta(x)$ of primes $p \leq x$ for which the hypothesis of Theorem 1.2 holds.

$(\bmod 4)$, we have $3 \mid B_{2}(n)=n\left(2^{p^{k-1}(p-1) / 2}-1\right)$ and so the following lemma completes the proof of Theorem 1.2.

Lemma 3.1. Suppose $n$ is with -1 . Let $\ell \nmid n$ be an odd prime with $\ell \mid B_{2}(n)$. Then $P(n) \mid B_{2}(n) / \ell$ if and only if $P(n) \mid B_{1}(n) / \ell$.

Proof. Let $m=\operatorname{ord}_{n}(2)$ so that $B_{2}(n)=n\left(2^{m / 2}-1\right)$. Since $\ell \mid B_{2}(n)$ and $\ell \nmid n$, we have $\ell \mid 2^{m / 2}-1$. Since $\ell$ is odd, $\ell \nmid 2^{m / 2}+1$. Let $v_{\ell}(x)$ denote the $\ell$-adic order of $x$. Then

$$
\begin{aligned}
v_{\ell}\left(B_{1}(n)\right)=v_{\ell}\left(2^{m}-1\right) & =v_{\ell}\left(\left(2^{m / 2}-1\right)\left(2^{m / 2}+1\right)\right) \\
& =v_{\ell}\left(2^{m / 2}-1\right)=v_{\ell}\left(n\left(2^{m / 2}-1\right)\right)=v_{\ell}\left(B_{2}(n)\right) .
\end{aligned}
$$

The result follows.

\section{Remarks}

As the example of $p=997$ shows, our argument requires 2 to remain prime in $\mathbb{Q}\left(\zeta_{n}\right)$. This means that 2 generates $(\mathbb{Z} / n \mathbb{Z})^{*}$ and so $n=p^{k}$ for some prime $p$. We must have $p \equiv 3$ or $5(\bmod 8)$, otherwise 2 is a square modulo $p$. Furthermore, we need $3 \mid B_{2}(n)$, which requires $p \equiv 1(\bmod 4)$. This explains the condition $p \equiv 5(\bmod 8)$.

We expect that there are infinitely many primes $p$ for which (1.1) has no odd solutions. Heuristically, we expect the fundamental unit to fall in each of the three nonzero residue classes modulo $p$ with equal probability, which suggests that these primes have density $1 / 3$ in the set of all primes $p \equiv 5(\bmod 8)$. The Generalised Riemann Hypothesis implies that the proportion of primes $p \equiv 5(\bmod 8)$ for which 2 is a primitive root is $A / 2$, where $A \approx 0.3739558$ is Artin's constant, as follows from the main result of [14]. Assuming that these two conditions on $p$ are independent, we thus expect that the primes satisfying the hypothesis of Theorem 1.2 have density $A / 6 \approx 0.0623259689$.

Numerically, we find that for primes up to $10^{9}$, this proportion is 0.0612819 , but this proportion creeps up as one considers ever larger upper bounds on $p$ (see Figure 1). This suggests that a Chebyshev bias-type phenomenon might be at work.

It is known that there are infinitely many squarefree integers $d \equiv 5(\bmod 8)$ for which the equation

$$
x^{2}-d y^{2}=4
$$


has no odd solutions (see [17]). (One can replace -4 by 4 in (1.1); this has the effect of merely squaring the fundamental unit).

Finally, our argument is related to that in [13]. That paper considers the same fields $K \subset L$ as we do, and uses the unit $N_{L / K}\left(1+\zeta_{n}\right) \in O_{K}^{*}$ to produce a relatively small solution to (1.1).

\section{Acknowledgement}

The author would like to thank the anonymous referee for useful comments and for pointing out the reference [14].

\section{References}

[1] V. I. Arnold, 'Complexity of finite sequences of zeroes and ones and geometry of finite spaces of functions', Funct. Anal. Other Math. 1 (2006), 1-15.

[2] A. Behn, C. Kribs-Zaleta and V. Ponomarenko, 'The convergence of difference boxes', Amer. Math. Monthly 112(5) (2005), 426-439.

[3] E. R. Berlekamp, 'The design of slowly shrinking labelled squares', Math. Comp. 29 (1975), 25-27.

[4] F. Breuer, 'Ducci sequences and cyclotomic fields', J. Difference Equ. Appl. 16(7) (2010), $847-862$.

[5] F. Breuer, E. Lötter and A. B. van der Merwe, 'Ducci sequences and cyclotomic polynomials', Finite Fields Appl. 13 (2007), 293-304.

[6] G. Brockman and R. J. Zerr, 'Asymptotic behaviour of certain Ducci sequences', Fibonacci Quart. 45(2) (2007), 155-163.

[7] M. Burmester, R. Forcade and E. Jacobs, 'Circles of numbers', Glasg. Math. J. 19 (1978), $115-119$.

[8] C. Ciamberlini and A. Marengoni, 'Su una interessante curiosità numerica', Periodiche di Matematiche 17 (1937), 25-30.

[9] A. Clausing, 'Ducci matrices', Amer. Math. Monthly 125(10) (2018), 901-921.

[10] H. Cohen, Number Theory, Volume I: Tools and Diophantine Equations, Graduate Texts in Mathematics, 239 (Springer, New York, 2007).

[11] A. Ehrlich, 'Periods of Ducci's N-number game of differences', Fibonacci Quart. 28(4) (1990), 302-305.

[12] B. Freedman, 'The four number game', Scripta Math. 14 (1948), 35-47.

[13] P. G. Hartung, 'On the Pellian equation', J. Number Theory 12(1) (1980), 110-112.

[14] P. Moree, 'On primes in arithmetic progression having a prescribed primitive root. II', Funct. Approx. Comment. Math. 39 (2008), 133-144.

[15] On-line Encyclopedia of Integer Sequences, entry \#A130229, https://oeis.org/A130229.

[16] G. J. Simmons, 'The structure of the differentiation digraphs of binary sequences', Ars Combin. 35A (1993), 71-88.

[17] P. Stevenhagen, 'On a problem of Eisenstein', Acta Arith. 74(3) (1996), 259-268.

[18] P. Zvengrowski, 'Iterated absolute differences', Math. Mag. 52(1) (1979), 36-40.

FLORIAN BREUER, University of Newcastle,

Callaghan, NSW 2308, Australia

e-mail: florian.breuer@newcastle.edu.au 